\title{
Minimal criteria for the identification of Gardnerella vaginalis isolated from the vagina
}

\author{
JLS JOLLY \\ From the Public Health Laboratory, Ipswich Hospital, Heath Road, Ipswich IP4 5PD
}

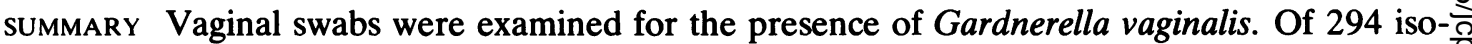
lates with appropriate colonial and cellular morphology subjected to an identification procedure, $\dot{\omega}$ $203(69 \%)$ were identified as $G$ vaginalis. The 91 isolates not identified as $G$ vaginalis were differentiated by their inability to ferment starch, cause diffuse $\beta$ haemolysis on human blood $A$ agar or hydrolyse hippurate. Other tests, often used in the identification of $G$ vaginalis, were $ळ$ found to be insufficiently specific. Failure to ferment starch coexisted with failure to cause $\beta$ 음 haemolysis and/or hydrolyse hippurate. The starch fermentation test may therefore be omitted. $\vec{\nabla}$ The tests for $\beta$ haemolysis and hippurate hydrolysis, being relatively simple to perform ando interpret, are considered indispensable for the accurate identification of $G$ vaginalis in the service laboratory.

The pathogenic significance of Gardnerella vaginalis is still in doubt. Since early work by Gardner and Dukes, ${ }^{1}$ numerous conflicting reports have appeared, with the result that clinicians and clinical laboratories alike hold differing opinions on the need to isolate and identify this organism. Difficulties in identifying $G$ vaginalis, and in defining the clinical condition associated with it, have hindered the clarification of its role. Accurate identification and exact definition is obviously important when searching for an association between organism and disease. Methods of bacterial identification are of little practical use if they are complex. Likewise shortened identification schemes are of little use if they fail to differentiate similar bacteria. In the past the methods most widely used to identify $G$ vaginalis have been those suggested by Dunkelberg ${ }^{2}$ which emphasise colonial morphology and fermentation tests. A dissecting microscope is required to examine the colonies and the fermentation tests are time-consuming and difficult to perform. More recent studies ${ }^{345}$ have suggested different identification schemes, in which the choice of criteria and complexity vary considerably. The purpose of this study was to evaluate selected criteria to ascertain the minimum number of tests needed by a routine laboratory for the identification of $G$ vaginalis.

Accepted for publication 1 December 1982

\section{Material and methods}

CULTURE

High vaginal swabs were submitted to the laboratory $\stackrel{\mathbb{2}}{2}$ from General Practitioners, family planning and $\vec{\circ}$ gynaecological clinics. The swabs, transported to the 3 laboratory in Stuart's transport medium, were cultured on chocolate agar (Columbia agar base? (Oxoid) with $10 \%$ horse blood heated to $80^{\circ} \mathrm{C}$ ), and증 Schaedler agar, (Oxoid) (with horse blood added:$(5 \%)$ ). Chocolate plates were incubated in $7 \% \mathrm{CO}_{2} 3$ in air for $48 \mathrm{~h}$ and the Schaedler plates for $48 \mathrm{~h}$ in an anaerobic environment. Films from small, smooth, entire colonies showing no haemolysis on chocolateo or Schaedler agars were Gram-stained. All small Gram-positive, -negative and variable pleomorphico bacilli were subjected to an identification procedure. Only predominant or abundant growths weres examined. The presence of other bacteria was alson noted. Presumed $G$ vaginalis, found as part of aN mixed bacterial flora, was subcultured on $\operatorname{starch}_{\sigma}^{\omega}$ serum agar. ${ }^{6}$ All specimens were also screened for Candida and Trichomonas.

IDENTIFICATION

Isolates were tested for starch fermentation by cul- $\frac{0}{-}$ ture on starch serum agar. This plate was also used疋 to detect inhibition by $3 \%$ hydrogen peroxide ${ }^{2}$ and catalase production; growth was scraped from the agar surface and touched onto the end of a capillary tube filled with $3 \%$ hydrogen peroxide. The evolu- $\bigcirc$ 
tion of bubbles was noted. Culture on human blood agar ${ }^{7}$ incubated in $7 \% \mathrm{CO}_{2}$ in air for $48 \mathrm{~h}$, revealed diffuse $\beta$ haemolysis. The rapid method of Hwang ${ }^{8}$ was used to test for hippurate hydrolysis. Disc tests for sensitivity determined by the comparative method, ${ }^{9}$ to sulphonamide $(100 \mu \mathrm{g})$, bacitracin (5 IU) and metronidazole $(50 \mu \mathrm{g})$ were done on Schaedler agar with lysed horse blood incubated anaerobically for $24 \mathrm{~h}$. Isolates of Gram-positive, -negative and variable small pleomorphic bacilli which showed starch fermentation, inhibition by $3 \%$ hydrogen peroxide, negative catalase reaction, ${ }^{2} \beta$ haemolysis on human blood agar, hippurate hydrolysis ${ }^{10}$ resistance to sulphonamide ${ }^{5}$ and sensitivity to bacitracin, ${ }^{10}$ were identified as $G$ vaginalis. Gram-positive, -negative and variable small pleomorphic bacilli failing to meet all of these criteria were called $G$ vaginalis-like organisms (GvLOs).

Haemophilus vaginalis NCTC 10287 served as the control organism.

Table 1 Collective features of 294 isolates.

\begin{tabular}{lcc}
\hline Characteristic & No & $(\%)$ \\
\hline Starch fermentation & 263 & $(90)$ \\
Catalase-negative & 294 & $(100)$ \\
Inhibition by $3 \%$ hydrogen peroxide & 294 & $(100)$ \\
$\beta$ haemolysis on human blood agar & 219 & $(75)$ \\
Hippurate hydrolysis & 251 & $(85)$ \\
Resistance to sulphonamide $(100 \mu \mathrm{g})$ & 294 & $(100)$ \\
Sensitivity to bacitracin $(5 \mathrm{IU})$ & 294 & $(100)$ \\
Sensitivity to metronidazole $(50 \mu \mathrm{g})$ & 259 & $(88)$ \\
\hline
\end{tabular}

Table 2 Isolates $(n=294)$ - reactions to three main criteria

\begin{tabular}{lllr}
\hline $\begin{array}{l}\text { Starch } \\
\text { fermentation }\end{array}$ & $\begin{array}{l}\text { Hippurate } \\
\text { hydrolysis }\end{array}$ & $\begin{array}{l}\beta \text { haemolysis } \\
\text { (human blood) }\end{array}$ & No \\
\hline+ & + & + & 203 \\
+ & + & - & 32 \\
+ & - & + & 15 \\
- & - & - & 13 \\
+ & - & - & 14 \\
- & + & + & 16 \\
- & + & + & 1 \\
- & + & -75 & 0 \\
Total -30 & -43 & +219 & 294 \\
Total +264 & +251 & & \\
\hline
\end{tabular}

\section{Results}

Two hundred and ninety-four isolates of Grampositive, -negative and variable small pleomorphic bacilli were examined; 203 (69\%) were subsequently identified as $G$ vaginalis.

\section{GRAM FILM}

Cellular morphology varied with the medium on which the organism was grown. Bacilli from chocolate agar were slightly larger and more pleomorphic than those from Schaedler agar. On this latter medium the morphology resembled the control organism (NCTC 10287) in being predominantly coccobacillary. The Gram reaction varied considerably with support medium and age of culture.

\section{IDENTIFICATION}

The features of the 294 isolates are shown in Table 1. All selected isolates were catalase-negative and were inhibited by $3 \%$ hydrogen peroxide, although the zone sizes varied. All isolates were resistant to sulphonamide and sensitive to bacitracin. The reaction patterns to starch fermentation, $\beta$ haemolysis and hippurate hydrolysis are shown in Table 2.

\section{Discussion}

In this study $G$ vaginalis was isolated satisfactorily on chocolate and Schaedler agars. Using these media other potential bacterial pathogens could be detected, so that additional isolation media were not necessary. The Gram stain reaction of selected isolates varied considerably with support media and age of culture. Consequently cellular morphology and size were more helpful in identification than the Gram reaction ifself. Indeed as the cell wall structure is typical of neither Gram-positive nor Gramnegative organisms, ${ }^{11}$ it seems inappropriate to adopt the Gram reaction as one of the identification criteria; that reaction was found to be of more use in excluding other bacteria than including possible $G$ vaginalis.

Gram-positive, -negative and variable small pleomorphic bacilli produced uniform colonial characteristics on the media used. Of 294 isolates examined all were found to be inhibited by $3 \%$ hydrogen peroxide, catalase-negative, resistant to sulphonamides and sensitive to bacitracin. As only 203 $(69 \%)$ isolates met other criteria for identification as $G$ vaginalis, these four tests seem insufficiently specific for routine identification of that organism. $259(88 \%)$ isolates were sensitive to metronidazole $(50 \mu \mathrm{g})$, but as in vitro resistance is reported to be a common feature of this organism ${ }^{31213}$ this test was excluded from the identification criteria. Separation of isolates into $G$ vaginalis and $G v$ LOs was based entirely upon starch fermentation, $\beta$ haemolysis on human blood agar and hippurate hydrolysis. Examination of these characteristics amongst the 294 isolates reveals that 44 isolates were negative for two or more characteristics and that no organism lysing human blood and hydrolysing hippurate failed to 
ferment starch. All isolates failing to ferment starch also failed to cause $\beta$ haemolysis and/or hydrolyse hippurate. Consequently given $\beta$ haemolysis and hippurate hydrolysis, the importance of which is obvious ${ }^{10}$ there seems little advantage in a starch fermentation test.

Fermentation of maltose and dextrose, in addition to starch have figured prominently in many identification procedures. ${ }^{14-17}$ However, Dunkelberg $^{18}$ has recently suggested that testing for acid production from dextrose and maltose in addition to starch is superfluous, an organism which acidifies starch being likely to acidify dextrose and maltose also. Ison et $a l^{4}$ have suggested an identification scheme that does not include fermentation tests. However it does not use the hippurate test, but relies on the catalase and oxidase tests. This study provides no evidence as to the usefulness of an oxidase test, but the catalase test was not found to be helpful. Another identification scheme suggested by Wells and $\mathrm{Goei}^{3}$ also dispenses with fermentation tests, and indeed with any subculture of isolates. Unfortunately, as a consequence, it relies heavily upon the recognition of characteristic colonial morphology, for which a dissecting microscope is necessary. In addition to this disadvantage it has also been sugested that the so-called characteristic colonial morphology is neither unique to, nor a feature of, all strains. ${ }^{18}$

The findings of this study suggest an identification scheme that relies upon two tests, $\beta$ haemolysis on human blood agar and hippurate hydrolysis. Both tests are simple to perform and easy to interpret and are therefore ideal for the service laboratory.

I wish to express my sincere thanks to Dr HWK Fell for his advice on the preparation of the manuscript. I am particularly grateful for the constructive criticisms made by Dr R Maskell and the persistent help and encouragement given to me by Dr JVT Gostling. Finally thanks to Mrs E Smith for typing the manuscript.

\section{References}

' Gardner HL, Dukes CD. Haemophilus vaginalis vaginitis. A newly defined specific infection previously classified "non- specific" vaginitis. Am J Obstet Gynaecol 1955;69:962-76.

"Dunkelberg WE, Skaggs R, Kellogg DS. Method for isolation and identification of Corynebacterium vaginale (Haemophilus vaginalis). Appl Microbiol 1970;19:47-52.

${ }^{3}$ Wells JI, Goei SH. Rapid identification of Corynebacterium vaginale in non-purulent vaginitis. J Clin Pathol 1981;34:917-20.

${ }^{4}$ Ison CA, Dawson SG, Hilton J, Csonka GW, Easmon CSF. Comparison of culture and microscopy in the diagnosis of Gardnerella vaginalis infection. J Clin Pathol 1982;35:55(1-4.

${ }^{5}$ Bailey RK, Voss JL, Smith RF. Factors affecting isolation and identification of Haemophilus vaginalis (Corynebacterium vaginale). J Clin Microbiol 1979;9:65-71.

- Islam AKMS. Starch serum agar-a differential medium for the isolation of Corynebacterium vaginale (Haemophilus vaginalis). J Clin Pathol 1977;30:291-2.

${ }^{7}$ Greenwood JR, Pickett MJ, Martin WJ, Mack EG. Haemophilus vaginalis (Corynebacterium vaginale): method for isolation and rapid biochemical identification. Health Lab Sci W 1977; 14:102-6.

${ }^{8}$ Hwang M, Ederer GM. Rapid hippurate hydrolysis method for presumptive identification of Grp B streptococci. J Clin Microbiol 1975;1:114-5.

${ }^{9}$ Stokes EJ, Waterworth PM. Antibiotic sensitivity tests by diffu- 윽 sion methods. ACP Broadsheet 55, 1966 (Revised 1972).

1" Greenwood JR, Pickett MJ. Salient features of Haemophilus vag- I inalis. J Clin Microbiol 1979;9:200-4.

" Greenwood JR, Pickett MJ. Transfer of Haemophilus vaginalis Gardner \& Dukes to a new genus Gardnerella: Gardnerella $\vec{\omega}$ vaginalis (Gardner \& Dukes) comb nov Int J Syst Bacteriol 1980;30: 170-8.

": Balsdon MJ, Taylor GE, Pead L, Maskell R, Corynebacterium vaginale and vaginitis: a controlled trial of treatment. Lancet 1980;i:501-5.

${ }^{13}$ Piot P, Van Dyck E, Goodfellow M, Falkow S, A taxonomic study of Gardnerella vaginalis (Haemophilus vaginalis), Gard- 응 ner \& Dukes 1955. J Gen Microbiol 1980;119:373-96.

${ }^{14}$ McCormack WM, Hayes CH, Rosner B, et al. Vaginal colonisation with Corynebacterium vaginale (Haemophilus vaginalis). $J$ 응 Infect Dis 1977;136 No 6:740-5.

15 Pheifer TA, Forsyth PS, Durfee MA, Pollock HM, Holmes KK. Nonspecific vaginitis. Role of Haemophilus vaginalis and 0 treatment with metronidazole. N Engl J Med 1978;298: 1429-34.

${ }^{16}$ Bramley HM, Dixon RA, Jones BM. Haemophilus vaginalis (Corynebacterium vaginale, Gardnerella vaginalis) in a family 3 planning clinic population. Br J Vener Dis 1981;57:62-6.

${ }^{17}$ Kinghorn GR, Jones BM, Chowdhury FH, Geary I. Balanopos- O thitis associated with Gardnerella vaginalis infection in men. Br J Vener Dis 1982;58:127-9.

18 Dunkelberg WE. Isolation and identification of Corynebacterium vaginale. J Am Med Technol 1978;40:9-11.

Requests for reprints to: JLS Jolly, Public Health ${ }^{N}$ Laboratory, Ipswich Hospital, Heath Road, Ipswich IP4 N 5PD, England. 